\title{
Anaplastic Lymphoma Kinase-Positive Anaplastic Large Cell Lymphoma with Cardiac Metastasis and Arterial Tumor Embolisms during First-Course Chemotherapy
}

\author{
Kozo Nagai $^{a} \quad$ Yukari Suyama $^{\mathrm{a}} \quad$ Daisuke Koga $^{\mathrm{a}} \quad$ Masanori Nishi $^{\mathrm{a}}$ \\ Chiaki Iida $^{a}$ Katsuya Tashiro $^{a} \quad$ Atsushi Danjo $^{b}$ Keita Kai $^{\mathrm{c}}$ \\ Muneaki Matsuo ${ }^{a}$ \\ ${ }^{a}$ Department of Pediatrics, Faculty of Medicine, Saga University, Saga, Japan; ${ }^{b}$ Department \\ of Oral and Maxillofacial Surgery, Faculty of Medicine, Saga University, Saga, Japan; \\ 'Department of Pathology, Saga University Hospital, Saga, Japan
}

\section{Keywords}

Anaplastic large cell lymphoma $\cdot$ Anaplastic lymphoma kinase $\cdot$ Heart metastasis $\cdot$ Arterial embolism

\begin{abstract}
We described an 11-year-old boy suffering from pediatric anaplastic lymphoma kinasepositive anaplastic large cell lymphoma with heart metastasis at diagnosis and arterial tumor embolisms during chemotherapy. Both the heart metastasis and pericardial effusion showed improvement with prednisolone, but numbness and pallor sequentially developed in his lower extremities during the first course of chemotherapy. Contrast-enhanced imaging revealed occlusion of the right anterior tibial artery and left popliteal artery. These symptoms were spontaneously remitted due to the compensation of other arteries. Arterial tumor embolism is a rare but possible complication when a lymphoma shows intracardiac infiltration.
\end{abstract}




\section{Case Reports in Oncology}

\section{Introduction}

Anaplastic large cell lymphoma (ALCL) is a T-cell non-Hodgkin lymphoma (NHL) that represents in about $3 \%$ of all adult NHLs and in $10-20 \%$ of pediatric NHLs [1]. In accordance with the World Health Organization classification, ALCL is composed of two main subtypes: anaplastic lymphoma kinase (ALK)-positive and ALK-negative [2]. ALK-positive ALCLs usually express the product of the nucleophosmin (NPM)-ALK chimeric gene, generated by the $\mathrm{t}(2 ; 5)$ chromosomal translocation between the $A L K$ gene on chromosome 2 and the NPM gene on chromosome 5. Positive rates of ALK tend to be higher (93\%) in pediatric ALCL patients [3]. It is recognized that ALK-positive ALCLs have a more favorable prognosis than ALK-negative ones [4], but the 2-year relapse rate among children and adolescents with ALCL still reaches $30 \%$ after intensive chemotherapy [5]. In addition, ALCLs with a small-cell or lymphohistiocytic $(\mathrm{SC} / \mathrm{LH})$ component are often diagnosed at advanced stages (stage III/IV) and associated with B symptoms: fever, night sweats, and weight loss. SC/LH ALCL has a relatively poor prognosis [6]. ALCL often develops in cervical or inguinal lymph nodes as well as in extranodular sites, such as skin, bone, soft tissue, liver, and lung; however, cases of ALCL with myocardial or pericardial involvement are rare.

We experienced a pediatric ALCL patient with heart metastasis and arterial tumor embolism complications during the first course of chemotherapy. Arterial tumor embolism is a rare but possible complication when a lymphoma shows intracardiac infiltration.

\section{Case Report}

An 11-year-old boy presented to a dental clinic with intermissive tooth pain and a mass in his left mandibular gingiva 2 months before hospital admission. The patient had no additionally significant medical or family history. On admission to our hospital, a 4-cm mass was observed in his oral cavity (fig. 1). He did not display fever, hepatosplenomegaly, or any other symptoms. Laboratory findings revealed inflammatory change (C-reactive protein, 4.71 $\mathrm{mg} / \mathrm{dl}$; immunoglobulin G, $1,770 \mathrm{mg} / \mathrm{dl}$; ferritin, $140.0 \mathrm{ng} / \mathrm{ml}$; IL-2R, 11,930 U/ml; erythrocyte sedimentation rate, $117 \mathrm{~mm} / \mathrm{h}$ ), normal blood cell count, normal transaminase levels, and normal blood coagulation tests. Chest X-ray showed mild cardiomegaly and a 6-cm extrathoracic mass. Systematic computed tomography (CT) scan revealed a $43 \times 25 \mathrm{~mm}$ osteolytic mass in the left submandibular bone (fig. 2a) and a $56 \times 46 \mathrm{~mm}$ mass in the left 3rd costal bone (fig. $2 \mathrm{~b}$ ). Furthermore, two masses (23 and $15 \mathrm{~mm}$ ) protruding into the left ventricular cavity and pericardial fluid were observed (fig. 2c, d). Electrocardiogram showed mild diffuse ST-elevation but no arrhythmia. Biopsy of the left 3rd costal bone mass was performed, and histopathologic examination showed small abnormal lymphocyte proliferation (fig. 2e). Large atypical lymphocytes (partially similar to Reed-Sternberg cells) were scattered in small numbers. Immunohistochemistry revealed the small abnormal lymphocytes to be positive for CD3, UCHL-1, Granzyme B, and CD56. CD30 and ALK were strongly positive in the large lymphocytes and weakly positive in the small lymphocytes (fig. 1g, fig. 2f). ALK was stained both in the cytosol and nucleus. Additionally, the large atypical lymphocytes were positive for epithelial membrane antigen (EMA) (fig. 2h). Based on the above observations, a diagnosis of small cell variant ALK-positive ALCL was made. Due to the presence of heart metastasis and pericardial effusion, prednisolone $30 \mathrm{mg} / \mathrm{m}^{2} /$ day for 5 days was administered prior to multidrug chemotherapy in accordance with the ALCL99 highrisk protocol $[7,8]$. After steroid therapy, the heart metastasis promptly decreased in size 


\section{Case Reports in Oncology}

and the pericardial effusion disappeared within a few days. First-course chemotherapy was started as scheduled without any heart complications; however, 4 days into chemotherapy, he developed numbness in his right leg. The numbness gradually worsened, and a few days later, he suffered from sudden pain and pallor in his lower right extremity after walking. Enhanced CT of his legs revealed an occlusion of the right anterior tibial artery. Collateral blood vessel extension was observed in the CT image, and symptoms became less severe on their own. Tumor embolism was initially suspected due to these symptoms, but thromboembolic complication could not be completely ruled out, and heparin was administered to prevent further complications. Despite this, a week later, the same complication developed in his left leg. As a measure to prevent serial thromboembolism, aspirin and beraprost sodium were then administered in addition to heparin. Magnetic resonance angiography showed occlusion of the left popliteal artery as well as the right anterior tibial artery (fig. 3). Symptoms were resolving, and conservative treatment was continued. No complications reignited, and antithrombogenic drugs were fully tapered off at 2 months. Re-perfusion of the occluded arteries has not been observed on follow-up MRI. The scheduled ALCL99 high-risk protocol was accomplished without any other severe complications. At the time of this publication (22 months after chemotherapy completion), the patient has been without disease in outpatient clinic follow-ups.

\section{Discussion}

The present case showed cardiac masses and pericardial effusion at the time of initial diagnosis, although, due to the absence of cardiac symptoms from lymphomatous cardiac infiltration and the presence of large concurrent mandibular gingival and left $3 \mathrm{rd}$ costal bone masses, this case does not meet the criteria for primary cardiac lymphoma.

Pediatric ALCL with cardiac involvement at diagnosis is rare. Currently, only 2 cases are known to have been reported: an 8-year-old primary cardiac ALCL patient [9] and a 2-yearold ALCL patient with cardiac tamponade [10]. Pediatric ALCL with cardiac involvement, like pediatric ALCL in general, seems to respond favorably to steroids and chemotherapy. Both cases were treated with the ALCL99 protocol and achieved long periods of complete remission. Cardiac tumors are most often seen in adulthood. Metastatic cardiac lymphomas are found in approximately $10-30 \%$ of lymphoma patients in autopsy studies [11], and these cases tend to involve cardiac dissemination at later stages of disease process. In pediatric cases, common primary tumors of heart metastasis are lymphoma, although neuroblastoma, Wilms' tumor, and malignant teratoma may sometimes give rise to cardiac metastasis [12]. When metastasis occurs, $75 \%$ of the cases involve the pericardium/epicardium, with most of them presenting as pericardial effusion [13]. Cardiac tumors can have various clinical presentations depending on the site of cardiac involvement. Patients can present with progressive heart failure, arrhythmia, chest pain, and syncope. The present case had neither arrhythmia nor heart failure.

To avoid methotrexate (MTX) toxicity with the ALCL99 high-risk protocol that includes high-dose MTX, pericardial effusion should be controlled by adequate pretreatment steroid administration. In this case, we administrated prednisolone before prephase treatment of ALCL99, and pericardial effusion completely disappeared during the prephase treatment. Thus, high-dose MTX was available from the first AM block of ALCL99.

Chemotherapy can cause tumor fragility via tissue necrosis, raising the risk of embolism. In the past, several cases of pulmonary tumor embolism arising from right atrial or ventricu- 
Nagai et al.: Anaplastic Lymphoma Kinase-Positive ALCL with Cardiac Metastasis and Arterial Tumor Embolisms during First-Course Chemotherapy

lar cardiac lymphoma have been reported $[14,15]$, whereas a cardiac lymphoma in the left ventricle presenting as aortic embolism is rare. In the present case, the cardiac lymphoma in the left ventricle was diminished in size by multidrug chemotherapy with tissue or tumor being set apart and circulating into peripheral arteries. Thereby, consecutive embolisms in both legs were caused during first-course chemotherapy, but have not been reignited after second-course chemotherapy completion. No changes in blood coagulation tests have been observed regarding these complications. If severe symptoms are caused by arterial embolism, embolic material should be removed accordingly by catheter intervention. In this case, the arterial embolisms did not occur at critical sites, and symptoms spontaneously remitted due to the compensation of other arteries.

In conclusion, physicians should consider the possibility of arterial embolism when treating lymphoma with left ventricular cavity invasion. To avoid this complication and drug-related side effects, gradual tumor reduction and improvement of pericardial effusion by steroid administration should be carried out prior to multidrug chemotherapy.

\section{Statement of Ethics}

Informed consent was obtained from the patient for the treatment and publishing of this case. The patient received treatment based on the chemotherapy protocol approved by our institutional protocol committee.

\section{Disclosure Statement}

The authors have no conflicts of interest to declare.

\section{References}

1 Wright D, McKeever P, Carter R: Childhood non-Hodgkin lymphomas in the United Kingdom: findings from the UK Children's Cancer Study Group. J Clin Pathol 1997;50:128-134.

2 Lim MS, de Leval L, Quintanilla-Martinez L: Commentary on the 2008 WHO classification of mature Tand NK-cell neoplasms. J Hematop 2009;2:65-73.

3 Brugières L, Deley MC, Pacquement $\mathrm{H}$, et al: $\mathrm{CD} 30(+)$ anaplastic large-cell lymphoma in children: analysis of 82 patients enrolled in two consecutive studies of the French Society of Pediatric Oncology. Blood 1998;92:3591-3598.

4 Gascoyne RD, Aoun P, Wu D, et al: Prognostic significance of anaplastic lymphoma kinase (ALK) protein expression in adults with anaplastic large cell lymphoma. Blood 1999;93:3913-3921.

-5 Rosolen A, Pillon M, Garaventa A, et al: Anaplastic large cell lymphoma treated with a leukemia-like therapy: report of the Italian Association of Pediatric Hematology and Oncology (AIEOP) LNH-92 protocol. Cancer 2005;104:2133-2140.

-6 Lamant L, McCarthy K, d'Amore E, et al: Prognostic impact of morphologic and phenotypic features of childhood ALK-positive anaplastic large-cell lymphoma: results of the ALCL99 study. J Clin Oncol 2011;29:4669-4676.

7 Le Deley MC, Rosolen A, Williams DM, et al: Vinblastine in children and adolescents with high-risk anaplastic large-cell lymphoma: results of the randomized ALCL99-vinblastine trial. J Clin Oncol 2010;28:3987-3993.

8 Wrobel G, Mauguen A, Rosolen A, et al: Safety assessment of intensive induction therapy in childhood anaplastic large cell lymphoma: report of the ALCL99 randomised trial. Pediatr Blood Cancer 2011;56:1071-1077.

$\checkmark 9$ Lauten M, Vieth S, Hart C, et al: Cardiac anaplastic large cell lymphoma in an 8-year old boy. Leuk Res Rep 2014;3:36-37. 


\section{Case Reports in Oncology}

\begin{tabular}{l|l}
\hline \multicolumn{2}{l}{ Case Rep Oncol 2016;9:440-446 } \\
\hline $10.1159 / 000447999$ & $\begin{array}{l}\text { (c) 2016 The Author(s). Published by S. Karger AG, Basel } \\
\text { www.karger.com/cro }\end{array}$ \\
\hline
\end{tabular}

Nagai et al.: Anaplastic Lymphoma Kinase-Positive ALCL with Cardiac Metastasis and Arterial Tumor Embolisms during First-Course Chemotherapy

-10 Mira-Perceval JG, Alcalá Minagorre PJ, Huertas Sánchez AM, et al: Cardiac tamponade associated with the presentation of anaplastic large cell lymphoma in a 2-year-old child. Case Rep Pediatr 2015;2015:487491.

11 Nascimento AF, Winters GL, Pinkus GS: Primary cardiac lymphoma: clinical, histologic, immunophenotypic, and genotypic features of 5 cases of a rare disorder. Am J Surg Pathol 2007;31:1344-1350.

12 Huh J, Noh CI, Kim YW, et al: Secondary cardiac tumor in children. Pediatr Cardiol 1999;20:400-403.

13 Ragland MM, Tak T: The role of echocardiography in diagnosing space-occupying lesions of the heart. Clin Med Res 2006;4:22-32.

14 Skalidis EI, Parthenakis FI, Zacharis EA, et al: Pulmonary tumor embolism from primary cardiac B-cell lymphoma. Chest 1999;116:1489-1490.

15 Kosugi M, Ono T, Yamaguchi H, et al: Successful treatment of primary cardiac lymphoma and pulmonary tumor embolism with chemotherapy. Int J Cardiol 2006;111:172-173.

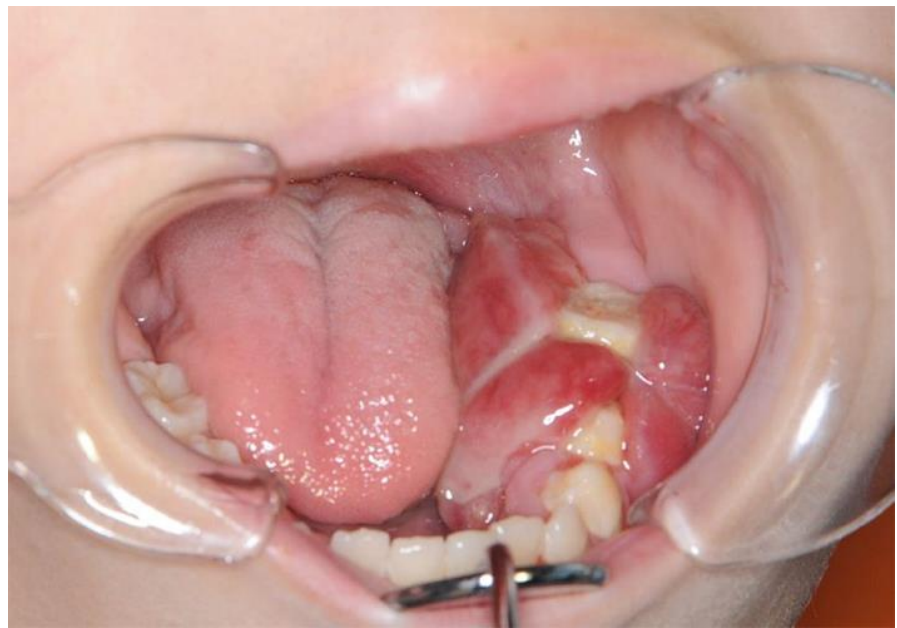

Fig. 1. Soft tissue mass in the left mandibular gingiva. 


\section{Case Reports in Oncology}

\begin{tabular}{l|l}
\hline Case Rep Oncol 2016;9:440-446 \\
\hline $10.1159 / 000447999$ & $\begin{array}{l}\text { (c) 2016 The Author(s). Published by S. Karger AG, Basel } \\
\text { www.karger.com/cro }\end{array}$ \\
\hline
\end{tabular}

Nagai et al: Anaplastic Lymphoma Kinase-Positive ALCL with Cardiac Metastasis and Arterial Tumor Embolisms during First-Course Chemotherapy
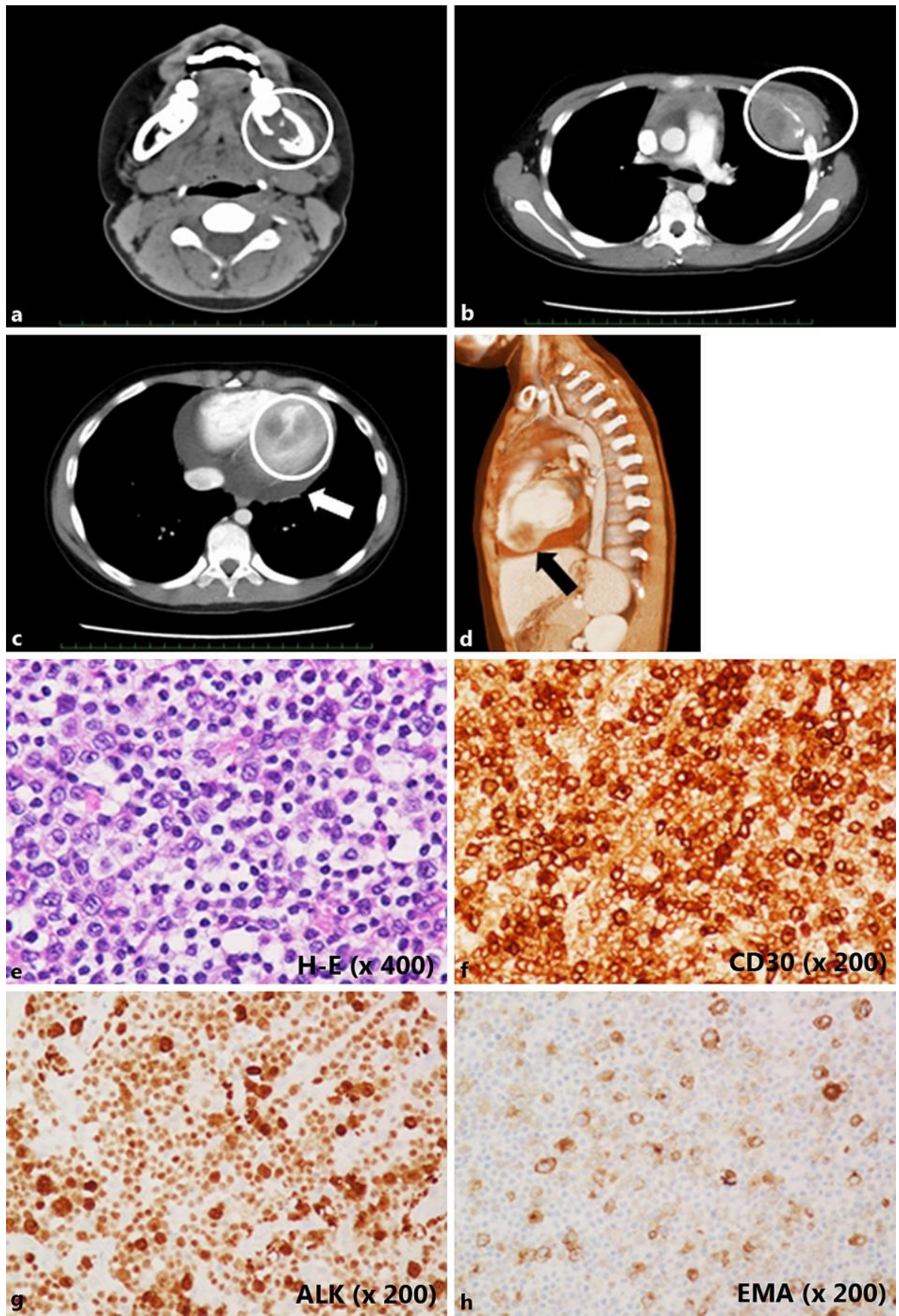

Fig. 2. CT imaging and histopathologic findings. Head CT scan before chemotherapy revealed a $43 \times 25 \mathrm{~mm}$ osteolytic mass in the left submandibular bone (a). Axial body CT image showed a $56 \times 46 \mathrm{~mm}$ osteolytic tumor in the left 3rd costal bone (b) and low-density tumor projecting into the left ventricular cavity (c). Sagittal reconstructed CT showed tumor projecting into the left ventricular cavity (d). Pericardial effusion was present. H-E staining of the left costal bone showed small abnormal lymphocytes predominantly proliferated and large atypical lymphocytes scattered (e). In immunohistochemical analysis, neoplastic cells were positive for CD30 (f). Large atypical cells were strongly ALK-positive, whereas small neoplastic cells were weakly positive (g). EMA staining showed large atypical cells to be positive for EMA immunoreactivity, while small cells were negative (h). Original magnification: $\times 400(\mathbf{e}) \times 200(\mathbf{f}-\mathbf{h})$. 


\section{Case Reports in Oncology}

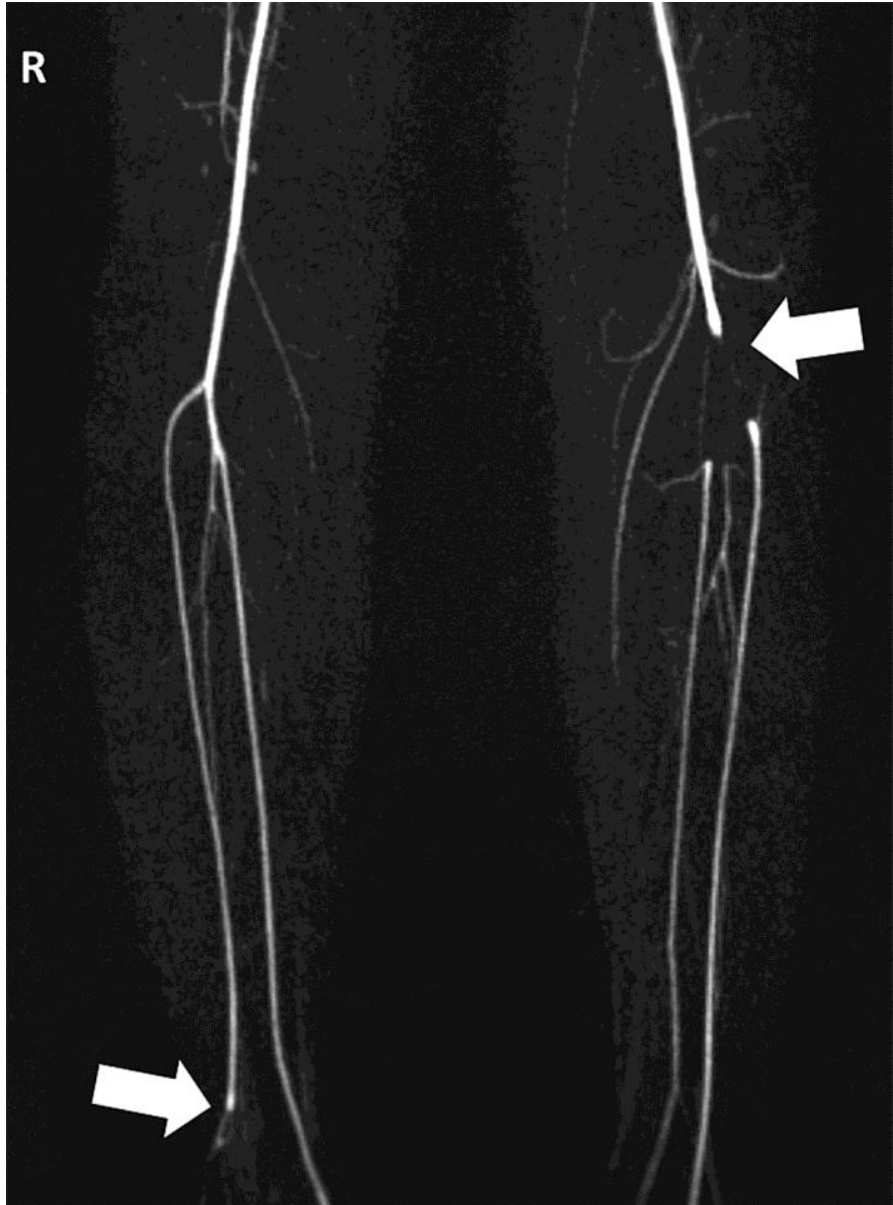

Fig. 3. Lower extremity magnetic resonance angiography after second embolic episode. Contrast-enhanced MRI revealed interruption of the left popliteal artery and right anterior tibial artery.

Nagai et al.: Anaplastic Lymphoma Kinase-Positive ALCL with Cardiac Metastasis and Arterial Tumor Embolisms during First-Course Chemotherapy

MR revealed interruption of the left popliteal artery and right anterior tibial artery. 